Research Article

\title{
Landing Trajectory Generation and Energy Optimization for Unmanned Lunar Mission
}

\author{
Md. Shofiqul Islam $\mathbb{I D}^{1}$ and Ibrahim M. Mehedi $\mathbb{D}^{1,2}$ \\ ${ }^{1}$ Department of Electrical and Computer Engineering (ECE), King Abdulaziz University, Jeddah 21589, Saudi Arabia \\ ${ }^{2}$ Center of Excellence in Intelligent Engineering Systems (CEIES), King Abdulaziz University, Jeddah 21589, Saudi Arabia \\ Correspondence should be addressed to Ibrahim M. Mehedi; imehedi@kau.edu.sa
}

Received 1 June 2021; Revised 12 June 2021; Accepted 20 June 2021; Published 1 July 2021

Academic Editor: Vijay Kumar

Copyright (C) $2021 \mathrm{Md}$. Shofiqul Islam and Ibrahim M. Mehedi. This is an open access article distributed under the Creative Commons Attribution License, which permits unrestricted use, distribution, and reproduction in any medium, provided the original work is properly cited.

\begin{abstract}
The moon is recognized as an important destination for space science and exploration. To find a satisfactory answer for the mystery of the universe and to make use of the lunar resources for the welfare of human beings, several space agencies are planning manned and unmanned missions on the moon. As a result, the concept of lunar vehicles has begun with an advanced descent scheme to execute a precise and safe landing on the surface of the moon. On the contrary, the energy budget is an important issue for any space mission. To reduce the cost of a space mission, it is necessary to design the vehicle trajectory based on optimized energy resources. Fuel is the main energy in a space mission. Therefore, a fuel-optimized energy generation technique is focused on this research. The design of an algorithm that generates a real-time trajectory for the descent and landing of a lunar probe is critical to ensuring a successful lunar landing mission. A scheme of dual-step trajectory generation for lunar descent is also investigated in this paper. In the algorithm developing process, the thrust-to-mass ratio is considered as a principle variable. Algorithm design along with mathematical modeling and simulation results are described in detail. In addition, the proposed method for generating reference trajectory profiles is also analyzed for fuel consumption and robustness.
\end{abstract}

\section{Introduction}

Since the moon is extensively observed as the first step from the Earth to space, numerous aerospace organizations have initiated several scientific operations towards the lunar expedition. Indeed, moon exploration and other planetary investigation concerns have acknowledged significant consideration from the 1960s [1-4]. Low-energy landing trajectory generation is an important issue for the upcoming space survey operations to accomplish different landing missions on planets, comets, and asteroids with numerous standards [5-10]. The likeness is that each operation needs a certain value of accurate and harmless landing competency specifically for the terrain consists of hazard to accomplish a sample return or survey missions in a scientifically attractive region. While the Apollo program is already too old, the lunar maria is not striking aims to investigate. A landing spot close to the principal peak of a large crater is believed to be the utmost technically fascinating target $[11,12]$. Such locations may provide us hints about the lunar origin because these areas are still totally unexplored. Self-governing landing spacecraft is a suitable candidate to explore the mentioned attractive areas on the moon, but undoubtedly, it is a hard and uncertain assignment to land near the scientifically interesting landing region. The main reason is the long communication delay because of the huge distance [13]. Consequently, the customary trajectory producing arrangements and deep space network-based GNC scheme is not appropriate for accurate and nonviolent moon landing, while every action from the powered descent towards terminal landing phases should be completed independently with the help of on-board electronics and software systems. This means that the spacecraft will be initially orbiting the moon with a certain orbital speed condition. From the 
orbital state, the spacecraft will initiate an automated powered descent phase generating a descent trajectory. The spacecraft will follow that trajectory towards the landing zone. In this process, GNC will have an active role to guide, navigate, and control the spacecraft. On the way of descent, the spacecraft may reproduce the trajectory to compensate for the error so that it flies towards the right destination. Until the terminal descent phase begins, this process will be carried out. The landing spacecraft should also be oriented in an upright position and softly touch the surface of the moon in order to ensure a soft landing $[14,15]$.

Figure 1 shows the principal task of the lunar guidance scheme in which the spacecraft is taken from horizontally oriented condition to almost vertical orientation. In this course of time, the speed of the spacecraft is transferred from the orbital condition to the vertical landing situation at a low speed. The essence of gravity-turn descent $[1,2,8,15,16]$ is included in the current investigation. Furthermore, it develops the solution towards a readily implementable algorithm. The direction of the thrust vector and the velocity vector is opposite to each other, and this is the principal technique of gravity-turn descent. The inertial measurement unit is used to identify the values of velocity and its direction vector. The attitude control system will use this information as an input for the purpose of maintaining the thrust vector opposite and parallel to the velocity direction. Actually, gravity-turn descent ensures upright landing with optimal fuel consumption. The plan and operation of spacecraft descent for lunar landing are separated into two different stages: the trajectory production as a reference flight path and the spacecraft guidance in real time. Escaping from the repeated long numerical operation and decreasing the difficulty for calculating the reference trajectory are very important issues for an on-board mission. Moreover, a universal reference trajectory must be produced so that the real-time guidance algorithm can use it properly. Before reference trajectory generation, it is essential to solving the dynamics of lunar landing spacecraft with different dimensions. Among those, a two-dimensional solution is described in [17]. Consequently, an upgraded 3-dimensional solution scheme is explained in [18]. A more detailed 3dimensional advanced resolution of the moon landing scheme is deliberated in [19]. DIDO optimization technique is also applied for the moon landing trajectory optimization scheme in [20]. To achieve a precise landing, an improved descent solution and a real-time applicable trajectory generation scheme are necessary. It should fulfill the goal of future missions so that it becomes highly robust and costeffective and can land safely. Reducing the complexity of developing the descent trajectories will certainly reduce the cost of preflight analysis and will increase the robustness for a precise lunar landing mission. Any scheme developed to replace the conventional schemes may have the same element structure: a descent solution element and a real-time applicable reference trajectory generating element. Solution methods of lunar descent available in the previous research studies are complex, iterative, and numerically calculated. Reference trajectory generation schemes followed the same style too. Moreover, some researchers implement preflight

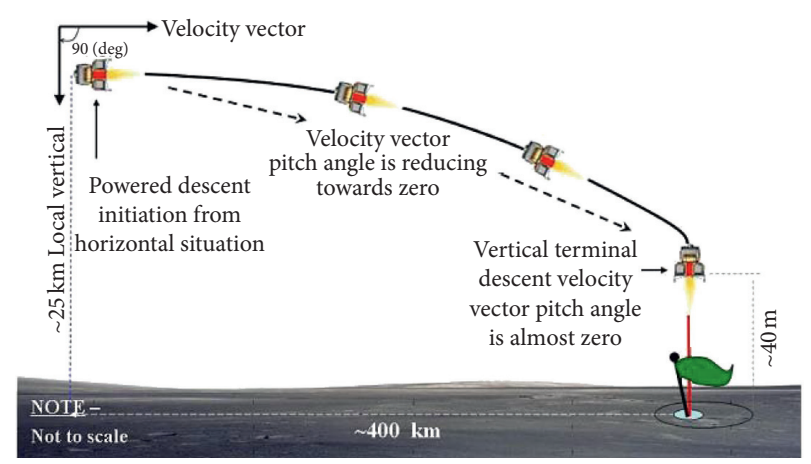

FIgURE 1: Typical lunar landing flight path. The horizontally oriented spacecraft is taken by lunar descent guidance algorithm. Initially, from orbital speed, the speed of spacecraft reduces to a very low speed condition, and it travels very long distance (several hundred kilometers) from the targeted landing area to the predefined landing zone. The spacecraft is in an almost vertical condition during landing.

reference trajectory generation techniques as well, which made the schemes poorly robust. Although a few literature studies investigated real-time reference trajectory generation schemes that are only for the portion of the terminal descent phase, again, a common limitation observed in all the previous research studies is that, after the earth-moon transfer, several complicated steps are included to reach the terminal descent initiation point, such as Hohmann transfer and de-orbit maneuver.

In this paper, we propose a qualitative solution to the equations for spacecraft speed, horizontal span, vertical range, and cross range as a function of velocity vector pitch angle and develop an analytical algorithm for dual-step reference trajectory generation. The new proposed scheme satisfies the vertical terminal landing condition and confirms a safe lunar landing mission. Entire descent solutions and comparisons are represented here for both two-dimensional and three-dimensional illuminations. Dual-step reference trajectory generation scheme is developed from equations obtained through the advanced lunar descent solution scheme to provide an accurate descent path starting from circular or elliptical parking orbit conditions. In addition, the robustness of the proposed reference trajectory generating algorithm is evaluated. Then, this paper proposes an advanced descent scheme and trajectory generation scheme for pin-point lunar landing. Analytical studies and simulations show the effectiveness and usefulness of the proposed schemes for a future lunar mission.

\section{Lunar Lander Dynamics}

A lunar descent schematic diagram is shown in Figure 2, where $L$ is shown for local vertical local horizontal (LVLH) reference frame. The relation between maneuver frame, $M$, and LVLH unit vectors is shown in this figure as well.

To land on the surface of the moon, it is now necessary to calculate the fundamental equations of motion. The surface of the moon is considered a uniform sphere-shaped body [1]. The dynamic equations of motion are divided into two parts. 


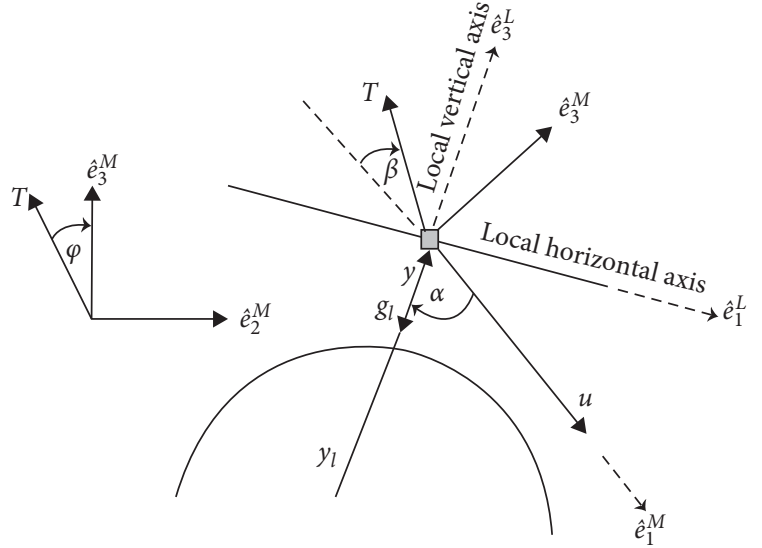

Figure 2: Lunar descent schematic diagram.

The first part describes the spacecraft dynamics and is given by the following equations:

$$
\begin{aligned}
\dot{u}(t) & =g_{l} \cos (\alpha)-N \cos (\beta), \\
\dot{\alpha}(t) & =\frac{1}{u}\left[\left(\frac{u^{2}}{y+y_{l}}-g_{l}\right) \sin \alpha-N \sin \beta \cos \varphi\right], \\
\dot{\psi}(t) & =\frac{1}{u \sin \alpha}[N \sin \beta \cos \varphi],
\end{aligned}
$$

where $u$ is the spacecraft velocity vector, $g_{l}$ is the lunar gravitational acceleration, $N$ is the ratio of thrust $F$ to the vehicle mass $m, \alpha$ is the pitch angle, $\beta$ is the thrust vector angle relative to the opposite of the velocity vector, $y$ is spacecraft altitude on the lunar surface, $y_{l}$ is the lunar radius, $\varphi$ is the thrust roll angle, and $\psi$ is the cross-range angle.

The second part describes the kinematics and is given by the following equations:

$$
\begin{aligned}
& \dot{y}(t)=-u \cos \alpha, \\
& \dot{x}(t)=u \sin \alpha \cos \psi \frac{y_{l}}{y+y_{l}}, \\
& \dot{c}(t)=u \sin \alpha \sin \psi \frac{y_{l}}{y+y_{l}} .
\end{aligned}
$$

Cross range and horizontal span are denoted by $x$ and $c$, respectively.

2.1. Preliminary Postulation. Spacecraft dynamic equations are needed to be transformed as a function of velocity vector pitch angle, $\alpha$. In this case, some necessary assumptions are made for thrust vector angle, thrust to mass ratio, and lunar gravitational acceleration force. $N$ (i.e., $F / m$ ) and $g_{l}$ are considered as constant where $\beta$ is set to zero. With these assumptions, equations (1) to (3) reduce to

$$
\begin{aligned}
& \dot{\alpha}(t)=\frac{1}{u}\left[\left(\frac{u^{2}}{y+y_{l}}-g_{l}\right) \sin \alpha\right], \\
& \dot{u}(t)=g_{l} \cos \alpha-N, \\
& \dot{\psi}(t)=0 .
\end{aligned}
$$

Therefore, $\psi(t)$ is constant. Since landing must take place close to the lunar surface, it is a reasonable practical assumption that $y \ll y_{l}$ which implies that $\left(y_{l} / y\right)+y_{l} \approx 1$. Using this in equations (5) and (6) gives

$$
\begin{aligned}
& \dot{x}(t)=u \sin \alpha \cos \psi, \\
& \dot{c}(t)=u \sin \alpha \sin \psi .
\end{aligned}
$$

\section{2D Advanced Numerical Solution}

In this advanced solution, an assumption for the centrifugal acceleration parameter is taken in a logical way. The ratio between lunar gravitational acceleration and centrifugal acceleration is defined by a constant value of $\Gamma$. Although it is a continuously varying parameter, it can be taken as a constant initially, and real-time guidance will rectify the errors caused by the environment. Therefore,

$$
\Gamma=\frac{u^{2} / y+y_{l}}{g_{l}},
$$

so

$$
\frac{u^{2}}{y+y_{l}}-g_{l}=-(1-\Gamma) g_{l}
$$

Considering the traditional lunar descent works $[1,8,16]$, the speed equation is obtained with the help the above assumption:

$$
\begin{aligned}
\dot{u}(\alpha) & =\frac{\dot{u}(t)}{\dot{\alpha}(t)}=u\left[\frac{g_{l} \cos \alpha-N}{-(1-\Gamma) g_{l} \sin \alpha}\right], \\
\frac{\mathrm{d} u}{u} & =\left[\frac{g_{l} \cos \alpha-N}{-(1-\Gamma) g_{l} \sin \alpha}\right] \mathrm{d} \alpha .
\end{aligned}
$$

Now, equation (15) is integrated to evaluate descent velocity:

$$
u(\alpha)=u_{0} e^{\int_{\alpha_{0}}^{\alpha}\left[g_{l} \cos \alpha-N /-(1-\Gamma) g_{l} \sin \alpha\right] \mathrm{d} \alpha} .
$$

If

$$
\int_{\alpha_{0}}^{\alpha}\left[\frac{g_{l} \cos \alpha-N}{-(1-\Gamma) g_{l} \sin \alpha}\right] \mathrm{d} \alpha=\ln \left[\frac{\sin \alpha}{\sin \alpha_{0}}\right]^{-1 /(1-\Gamma)}+\ln \left[\frac{\tan \left(\alpha_{0} / 2\right)}{\tan (\alpha / 2)}\right]^{-N /(1-\Gamma) g_{l}}
$$


then

$$
u(\alpha)=u_{0}\left[\frac{\sin \alpha}{\sin \alpha_{0}}\right]^{-1 /(1-\Gamma)}\left[\frac{\tan \left(\alpha_{0} / 2\right)}{\tan (\alpha / 2)}\right]^{-N /(1-\Gamma) g_{l}},
$$

as $\tan (\alpha / 2)=(1-\cos \alpha / \sin \alpha)$, and it is assumed that $\tau=$ $1 / 1-\Gamma$ and $\rho=N / g_{l}$, where $\rho>0$ so that

$$
u(\alpha)=u_{0}\left[\frac{\sin \alpha}{\sin \alpha_{0}}\right]^{-\tau}\left[\frac{\left(1-\cos \alpha_{0} / \sin \alpha_{0}\right)}{(1-\cos \alpha / \sin \alpha)}\right]^{-\tau \rho} \text {. }
$$

Here, the centrifugal acceleration parameter is measured by $\tau=1 / 1-\Gamma$. Therefore, the speed equation is obtained:

$$
u(\alpha)=u_{0}\left(\frac{\sin \alpha}{\sin \alpha_{0}}\right)^{-\tau(1+\rho)}\left(\frac{1-\cos \alpha_{0}}{1-\cos \alpha}\right)^{-\tau \rho}
$$

Now, vertical range $y(\alpha)$, time to descent $t_{D}$, and horizontal span $x(\alpha)$ are defined as follows:

$$
\dot{t}_{D}=\frac{1}{\dot{\alpha}(t)}=\frac{-\tau u(\alpha)}{g_{l} \sin \alpha}=\frac{-\tau u_{0}}{g_{l}} \frac{\left(1-\cos \alpha_{0}\right)^{-\tau \rho}}{\left(\sin \alpha_{0}\right)^{-\tau(1+\rho)}} \frac{(\sin \alpha)^{-\tau(1+\rho)-1}}{(1-\cos \alpha)^{-\tau \rho}} \text {. }
$$

Then,

$$
\dot{t}_{D}=G_{t_{D}} \frac{(\sin \alpha)^{-\tau(1+\rho)-1}}{(1-\cos \alpha)^{-\tau \rho}}
$$

where

$$
G_{t_{D}}=\frac{-\tau u_{0}}{g_{l}} \frac{\left(1-\cos \alpha_{0}\right)^{-\tau \rho}}{\left(\sin \alpha_{0}\right)^{-\tau(1+\rho)}} .
$$

For vertical range or altitude,

$$
\dot{y}(\alpha)=\frac{\dot{y}(t)}{\dot{\alpha}(t)}=-\frac{-\tau u(\alpha)^{2} \cos \alpha}{g_{l} \sin \alpha},
$$

then

$$
\dot{y}(\alpha)=\frac{\tau u_{0}^{2}}{g_{l}} \frac{\left(1-\cos \alpha_{0}\right)^{-2 \tau \rho}}{\left(\sin \alpha_{0}\right)^{-2 \tau(1+\rho)}} \frac{(\sin \alpha)^{-2 \tau(1+\rho)} \cos \alpha}{(1-\cos \alpha)^{-2 \tau \rho} \sin \alpha},
$$

so

$$
\dot{y}(\alpha)=G_{y} \frac{(\sin \alpha)^{-2 \tau(1+\rho)-1} \cos \alpha}{(1-\cos \alpha)^{-2 \tau \rho}},
$$

where

$$
G_{y}=\frac{\tau u_{0}^{2}}{g_{l}} \frac{\left(1-\cos \alpha_{0}\right)^{-2 \tau \rho}}{\left(\sin \alpha_{0}\right)^{-2 \tau(1+\rho)}} .
$$

For horizontal span or down range,

$$
\dot{x}(\alpha)=\frac{\dot{x}(t)}{\dot{\alpha}(t)}=-\frac{-\tau u(\alpha)^{2} \sin \alpha}{g_{l} \sin \alpha},
$$

then

$$
\dot{x}(\alpha)=\frac{-\tau u_{0}^{2}}{g_{l}} \frac{\left(1-\cos \alpha_{0}\right)^{-2 \tau \rho}}{\left(\sin \alpha_{0}\right)^{-2 \tau(1+\rho)}} \frac{(\sin \alpha)^{-2 \tau(1+\rho)}}{(1-\cos \alpha)^{-2 \tau \rho}},
$$

so

$$
\dot{x}(\alpha)=G_{x} \frac{(\sin \alpha)^{-2 \tau(1+\rho)}}{(1-\cos \alpha)^{-2 \tau \rho}},
$$

where

$$
G_{x}=\frac{-\tau u_{0}^{2}}{g_{l}} \frac{\left(1-\cos \alpha_{0}\right)^{-2 \tau \rho}}{\left(\sin \alpha_{0}\right)^{-2 \tau(1+\rho)}}
$$

As a precaution, we recommend taking an integer value for $\tau$ in order to avoid fractional integration. On the contrary, the value of $\Gamma$ can be set by a suitable fractional value so that $\tau$ becomes purely an integer quantity. However, the authors observed that the best choice is to fix some direct integer values for $\tau$ which prove more logical in approximation. Therefore, some test values $(1,2,3,4, \ldots)$ for $\tau$ are used in the above equations while integrating. Other necessary parameters are approximated by constant values such as $N=4[\mathrm{~N} / \mathrm{kg}]$ and $g_{l}=1.623\left[\mathrm{~m} / \mathrm{s}^{2}\right]$. The range of the velocity vector pitch angle $\alpha$ is considered between 90 [deg] and 0 [deg]. The starting speed of descent is chosen as the orbital speed, $1688[\mathrm{~m} / \mathrm{s}]$.

Computer simulation results are produced to show the influence of $\tau$ in Figures 3(a)-3(e). The figures show the trajectory of vertical range, speed, horizontal span, and time of lunar landing spacecraft in powered descent. These figures demonstrate comparative performance when the dynamics equations are compared with traditional methods. It can be mentioned that traditional solutions do not consider the estimation of the centrifugal acceleration parameter. In these simulation results, the impact of varying $\tau$ on spacecraft vertical range, speed, horizontal span, and descent time has been observed. It has been found that the vertical range is mostly influenced in this case. An assessment is conducted on simulation results to choose a suitable value of $\tau$. Consequently, improved responses are found for advanced solution if $\tau=2$.

\section{3D Numerical Solution}

To find a numerical solution during the powered descent phase, the above equations need to be simplified and rearranged in a format suitable for a numerical solver. We derive below the equations for speed $u$, time $t$, downrange $x$, altitude $y$, and cross-range $c$ as a function of a single variable, namely, the velocity vector pitch angle $\alpha$.

Using equations (7) and (8), the equation for speed $u$ is derived as follows:

$$
\dot{u}(\alpha)=\frac{\mathrm{d} u / \mathrm{d} t}{\mathrm{~d} \alpha / \mathrm{d} t}=\frac{\left(g_{l} \cos \alpha-N\right)}{(1 / u)\left(\left(u^{2} / y_{l}\right)-g_{l}\right) \sin \alpha} .
$$

Then, 


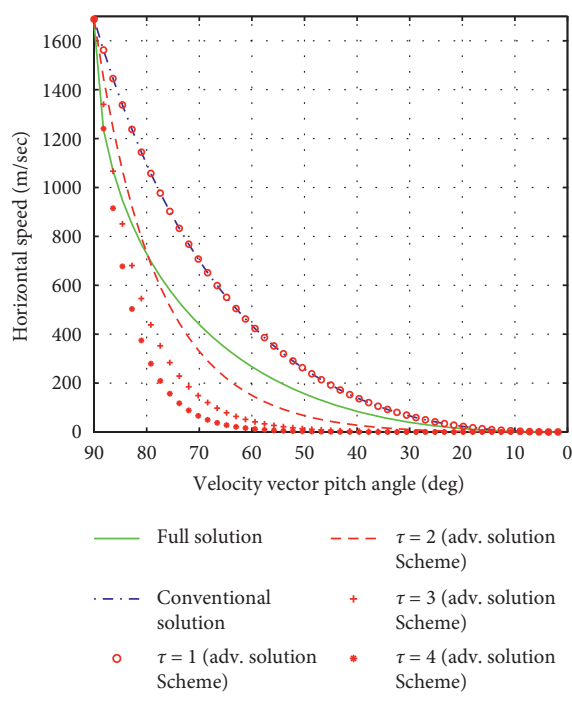

(a)

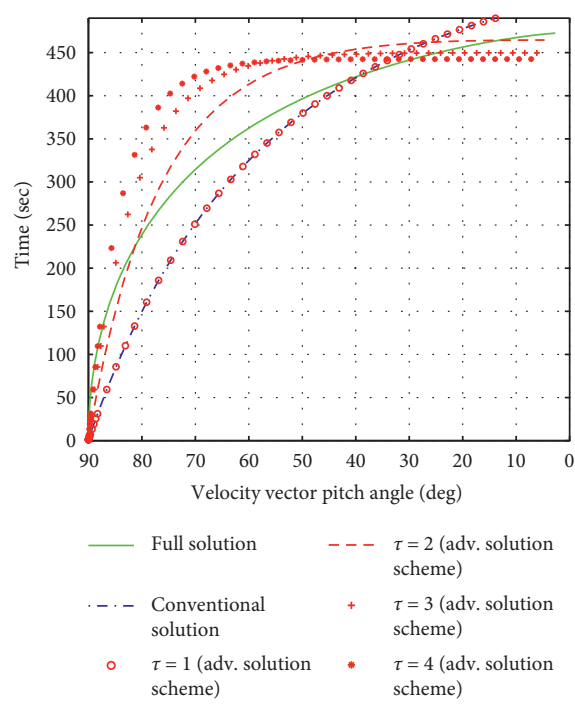

(c)

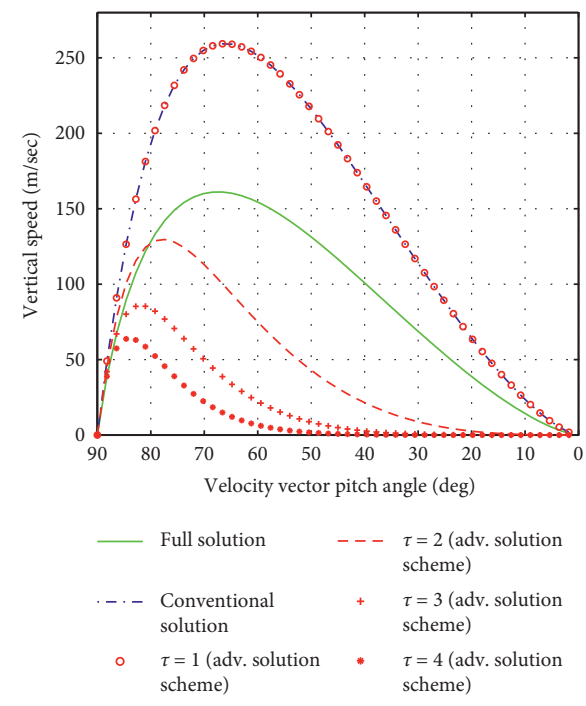

(b)

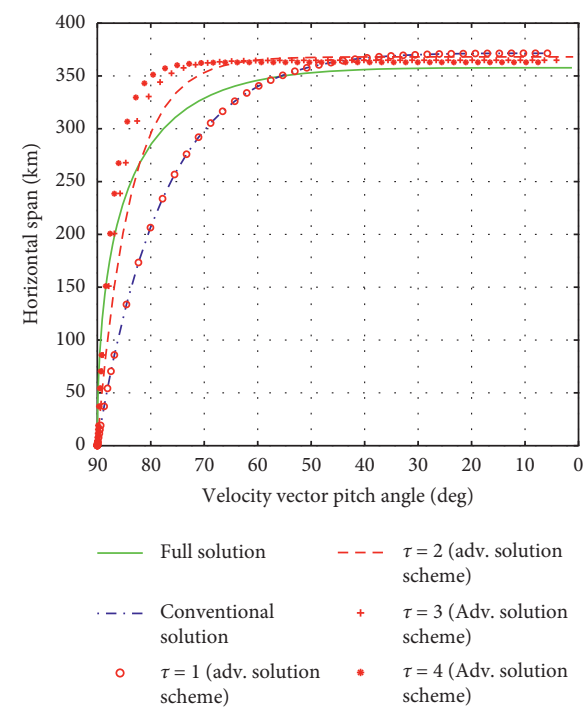

(d)

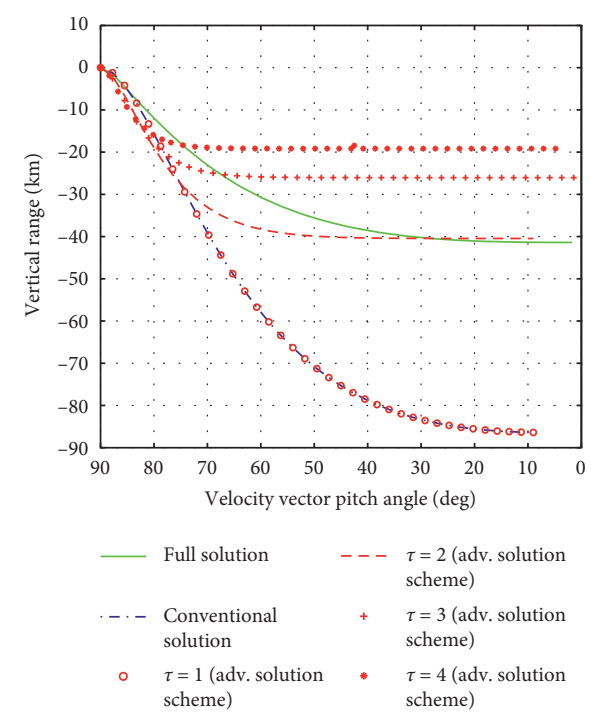

(e)

Figure 3: Comparison of advanced solution to fully integrated numerical solution and conventional solution. (a) Comparison-horizontal speed. (b) Comparison-vertical speed. (c) Comparison-time. (d) Comparison-horizontal span. (e) Vertical range. 


$$
\frac{1}{u}\left(\frac{u^{2}}{y_{l}}-g_{l}\right) \mathrm{d} u=\frac{g_{l} \cos \alpha-N}{\sin \alpha} \mathrm{d} \alpha .
$$

This can be integrated as

$$
\int_{u_{0}}^{u} \frac{1}{u}\left(\frac{u^{2}}{y_{l}}-g_{l}\right) \mathrm{d} u=\int_{\alpha_{0}}^{\alpha} \frac{g_{l} \cos \alpha-N}{\sin \alpha} \mathrm{d} \alpha .
$$

Therefore, the equation for speed becomes

$$
u(\alpha)=\left[-g_{l} y_{l} W\right]^{1 / 2},
$$

where the Lambert function $W$ is expressed as

$$
W=-\frac{u_{0}^{2}}{g_{l} y_{l}} \frac{\sin \alpha^{-2}}{e^{\left(u_{0}^{2} / g_{l} y_{l}\right)}}\left(\frac{1+\cos \alpha}{\sin \alpha}\right)^{-\left(2 N / g_{l}\right)} .
$$

Using equations (8) and (12), the descent time $t_{D}$ can be obtained by integrating the following equation expressed as a function of $\alpha$ :

$$
\dot{t}_{D}(\alpha)=\frac{\mathrm{d} t_{D}}{\mathrm{~d} \alpha}=\frac{\mathrm{d} u / \mathrm{d} \alpha}{\mathrm{d} u / \mathrm{d} t_{D}}=\frac{\dot{u}(\alpha)}{\dot{u}(t)} .
$$

Similarly, the equation for altitude can be rewritten as

$$
\dot{y}(\alpha)=\frac{\mathrm{d} y}{\mathrm{~d} \alpha}=\frac{\mathrm{d} y}{\mathrm{~d} t_{D}} \frac{\mathrm{d} t_{D}}{\mathrm{~d} \alpha}=\dot{y}(t) \dot{t}_{D}(\alpha) .
$$

Now, substituting the values from equation (4) gives

$$
\dot{y}(\alpha)=-u \cos \alpha \dot{t}_{D}(\alpha),
$$

where $u$ can be replaced from equation (13). Similar procedure can be followed to express the horizontal span as a function of the velocity vector pitch angle $\alpha$ :

$$
\dot{x}(\alpha)=\frac{\mathrm{d} x}{\mathrm{~d} \alpha}=\frac{\mathrm{d} x}{\mathrm{~d} t_{D}} \frac{\mathrm{d} t_{D}}{\mathrm{~d} \alpha}=\dot{x}(t) \dot{t}_{D}(\alpha) .
$$

Using equation (10) gives

$$
\dot{x}(\alpha)=u \sin \alpha \cos \psi \dot{t}_{D}(\alpha) .
$$

A similar procedure is applied to derive the following equation for the cross range:

$$
\dot{c}(\alpha)=u \sin \alpha \sin \psi \dot{t}_{D}(\alpha) .
$$

\section{Three-Dimensional Advanced Descent Solution}

The governing equations for a 3-dimensional advanced descent scheme are solved in the same manner as the 2dimensional scheme described in Section 3. The only additional aspect is to include the solution of the cross-range dynamic equation of lunar landing spacecraft in this $3 \mathrm{D}$ advanced descent solution. Therefore, it is necessary to represent the cross-range equation as a function of the velocity vector pitch angle. The remaining assumptions are the same as those described in the $2 \mathrm{D}$ scheme. Therefore, for cross-range,

$$
\begin{aligned}
\dot{c}(\alpha) & =\frac{\dot{c}(t)}{\dot{\alpha}(t)}=\frac{-\tau u_{0}^{2}}{g_{l}} \frac{\left(1-\cos \alpha_{0}\right)^{-2 \tau \rho}}{\left(\sin \alpha_{0}\right)^{-2 \tau(1+\rho)}} \sin \psi \frac{(\sin \alpha)^{-2 \tau(1+\rho)}}{(1-\cos \alpha)^{-2 \tau \rho}} \\
& =G_{c} \frac{(\sin \alpha)^{-2 \tau(1+\rho)}}{(1-\cos \alpha)^{-2 \tau \rho}} \sin \psi,
\end{aligned}
$$

where $G_{c}=\left(-\tau u_{0}^{2} / g_{l}\right)\left(\left(1-\cos \alpha_{0}\right)^{-2 \tau \rho} /\left(\sin \alpha_{0}\right)^{-2 \tau(1+\rho)}\right)$.

5.1. Three-Dimensional Response. Based on that derivation, a computer simulation is performed. Figure 4 represents a comparison of 3-dimensional trajectory responses for spacecraft descent on the lunar surface, while the governing equations are solved by complete integration method, conventional illumination, and advanced solution scheme. Equations of different states are numerically integrated with the constant values for lunar gravitational acceleration $g_{l}$, thrust to mass ratio $N$, initial vehicle speed $u_{0}$, and initial velocity vector pitch angle $\alpha_{0}$ mentioned in Table 1 . Simulation is categorized for different values of crossing angle between 5 degrees and 20 degrees with an increment of 5 degrees. It has been observed that altitude is not affected by changes in crossing angle. On the contrary, down range and cross-range distances are influenced because of different values of crossing angle. Results show that the trajectory response of less complex advanced solutions is always following the response of ideal but complex numerical solutions and having better performance than the conventional method of solution.

\section{Study on Fuel Consumption}

It is known that if the fuel is burnt or the thrusters are ignited, the mass of the vehicle decreases. So, the mass flow equation is shown in equation (44). The subscripts " $E$ " denotes for engine and " $J$ " distinguishes reaction control system (RCS) jet quantities:

$$
\dot{m}=-\frac{T_{\max E}}{V_{e x E}}-\frac{T_{\max J}}{V_{e x J}} \sum_{j=1}^{N_{j}} K_{j}
$$

Neglecting the thrusters, a new mathematical mass flow equation is shown in equation (45), which is similar to equation (44) without the thrust action. It is a suitable assumption because the mass flow due to RCS jets is much smaller than that due to the main engine:

$$
\dot{m}=-\frac{T_{\max E}}{V_{\text {exE }}} .
$$

The equation for descent time is found as a function of velocity vector pitch angle, $\alpha$ :

$$
\dot{t_{D}}(\alpha)=\frac{\mathrm{d} t_{D}}{\mathrm{~d} \alpha}
$$




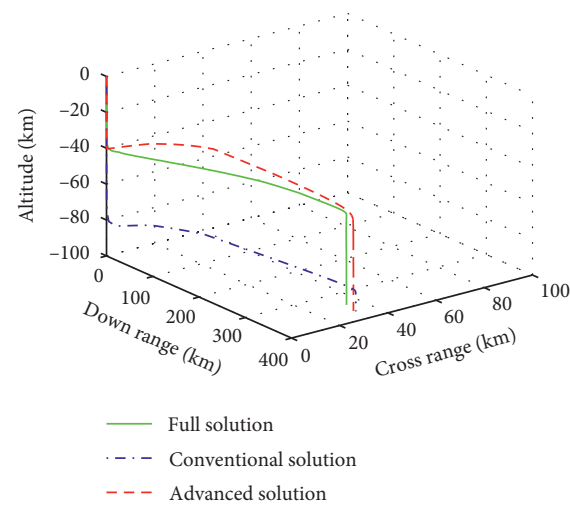

(a)

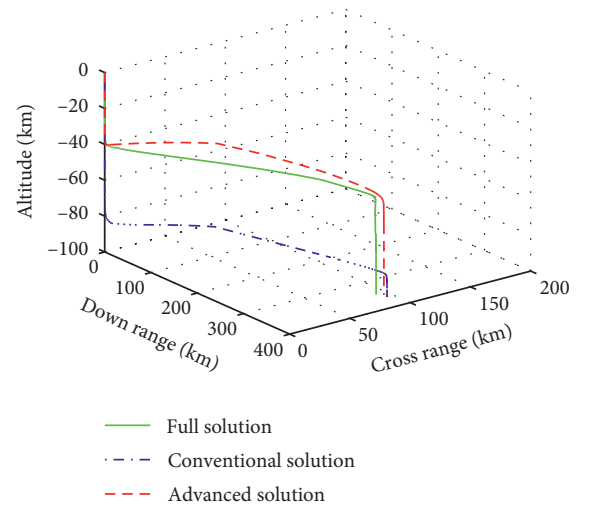

(c)

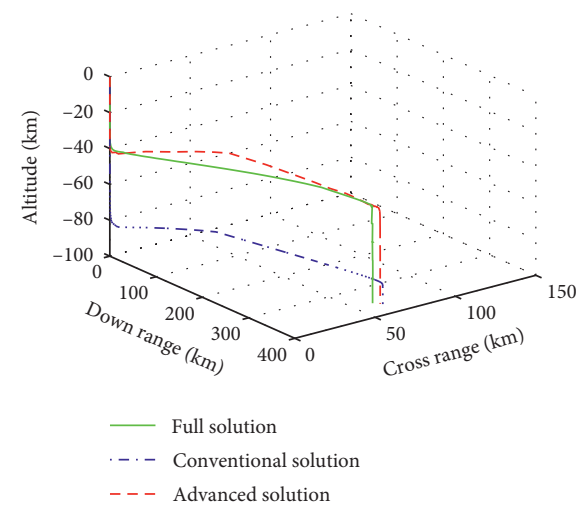

(b)

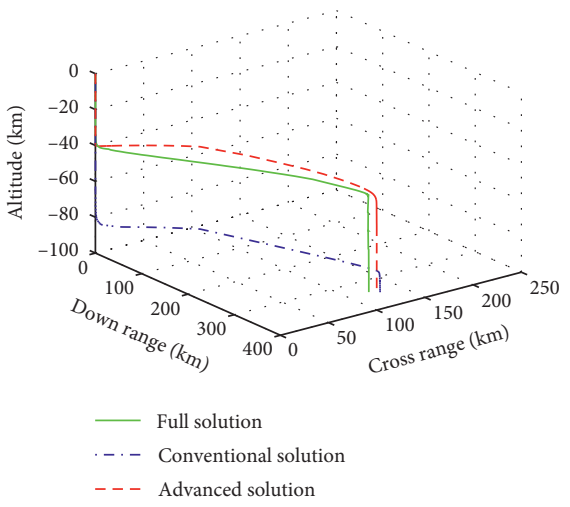

(d)

Figure 4: Three-dimensional flight path comparison between numerical and analytical solution for different crossing angle. (a) Crossing angle $=5.0$ deg. (b) Crossing angle $=10.0 \mathrm{deg}$. (c) Crossing angle $=15.0 \mathrm{deg}$. (d) Crossing angle $=20.0 \mathrm{deg}$.

TABle 1: Parameters of uncertainty for robustness analysis.

\begin{tabular}{lcccc}
\hline Initial velocity $(\mathrm{m} / \mathrm{s})$ & Final velocity $(\mathrm{m} / \mathrm{s})$ & $\begin{array}{c}\text { Initial velocity vector pitch } \\
\text { angle } \leq 90(\mathrm{deg})\end{array}$ & $\begin{array}{c}\text { Initial velocity vector pitch } \\
\text { angle } \geq 90(\mathrm{deg})\end{array}$ & $\begin{array}{c}\text { Lunar gravity } \\
\text { ang }\end{array}$ \\
\hline 1620 & 10 & 87.5 & 90.0 & 90.5 \\
1650 & 20 & 88.0 & 91.0 & $g_{l}+0.1$ \\
1680 & 30 & 88.5 & 91.5 & $g_{l}+0.2$ \\
1710 & 40 & 89.0 & 92.0 & $g_{l}+0.3$ \\
1740 & 50 & 89.5 & 92.5 & $g_{l}+0.4$ \\
1770 & 60 & 90.0 & $g_{l}+0.5$ \\
\hline
\end{tabular}

The fuel mass flow rate is resolved in a manner identical to the advanced solution proposed in Section 5 . With the help of equations (22), (45), and (46), vehicle mass flow is obtained:

$$
\dot{m}(\alpha)=\frac{\mathrm{d} m}{\mathrm{~d} t} \frac{\mathrm{d} t}{\mathrm{~d} \alpha}=-\frac{T_{\max E}}{V_{\text {exE }}} G_{t_{D}} \frac{(\sin \alpha)^{-2(1+\rho)-1}}{(1-\cos \alpha)^{-2 \rho}},
$$

where

$$
\begin{aligned}
G_{t_{D}} & =\frac{-2 u_{0}}{g_{l}} \frac{\left(1-\cos \alpha_{0}\right)^{-2 \rho}}{\left(\sin \alpha_{0}\right)^{-2(1+\rho)}}, \\
\rho & =\frac{N}{g_{l}} .
\end{aligned}
$$

Fuel consumption of the proposed dual-step reference trajectory generation is discussed in this section. Simulated fuel usage scenarios are shown in Figures 5-7 for N1, N2, and also combined. Maximum engine thrust and engine exhaust velocity are considered as $8000 \mathrm{~N}$ and $3500 \mathrm{~m} / \mathrm{s}$, respectively, for computer simulation while estimating fuel consumption during spacecraft powered descent phase implementing the proposed scheme. It is already mentioned that the output of the proposed algorithm is to produce a reference trajectory space in terms of a matrix containing all possible values of $N 1$ and $N 2$ for different destinations. For all the possible paths of descent to the particular destinations, estimation of fuel consumption can be done. For simplicity, in this section, one example is discussed while the spacecraft starts descent under $100 \mathrm{~km}$ circular parking orbit condition. 


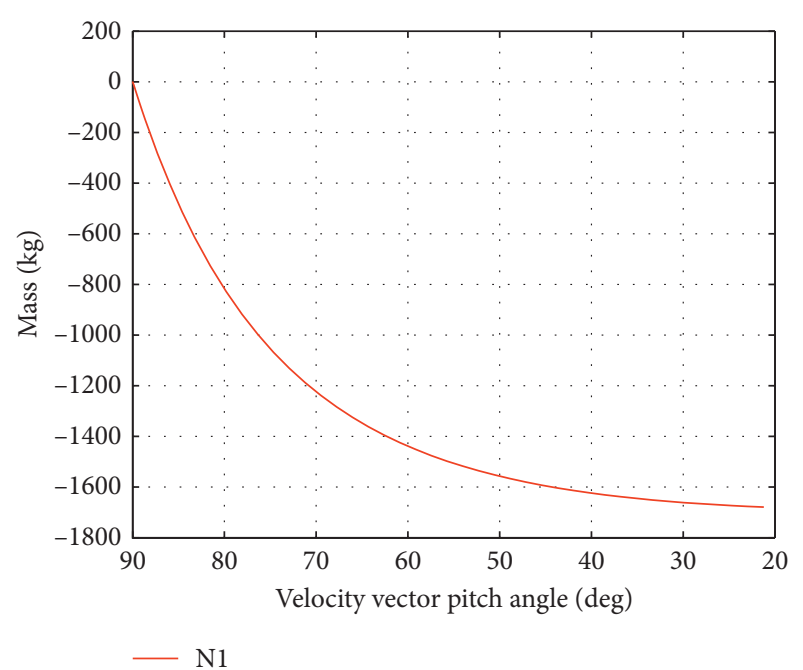

Figure 5: Fuel consumption during $N 1$.

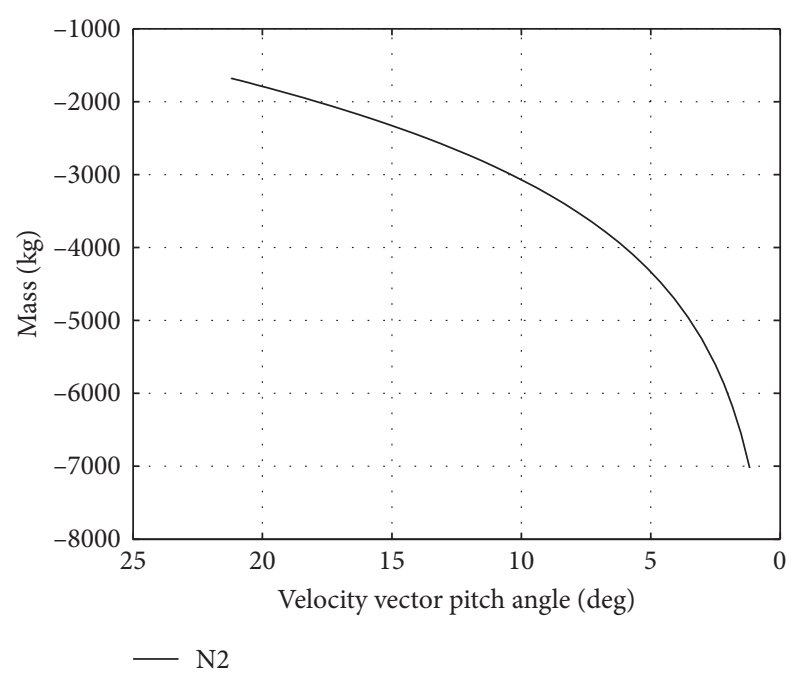

FIGURE 6: Fuel consumption during N2.

For example, under $100 \mathrm{~km}$ circular parking orbit conditions, the spacecraft needs to travel $415 \mathrm{~km}$ in horizontal direction to reach the predefined destination. For this purpose, the spacecraft needs to choose the exact combination of $N 1$ and $N 2$. The author found $N 1=3.6$ and $\mathrm{N} 2=1.6$ from the output matrix of the proposed algorithm. Figures 5-7 show the estimation of fuel consumption by the proposed dual-step reference trajectory generation method.

\section{Study on Robustness}

The initial conditions and the system parameters of the landing module may have uncertainties. Therefore, a study of robustness is useful. Navigation and attitude errors are considered for the study of robustness for the proposed dualstep trajectory generation scheme. Sensitivities to initial and final navigation errors and sensor errors to determine the vehicle attitude are evaluated.

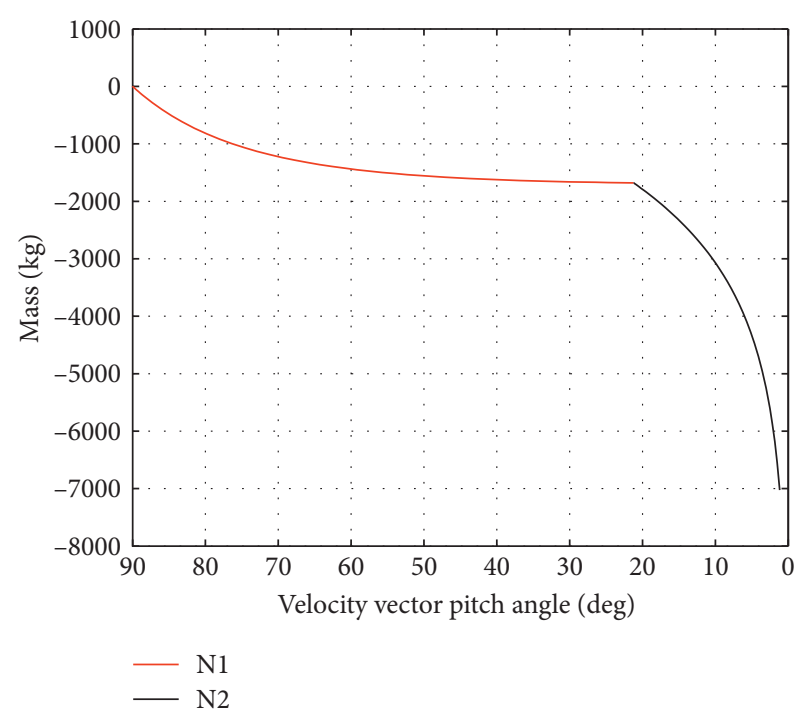

Figure 7: Fuel consumption during N1and N2.

Due to the navigation sensor errors, the initial velocity of the lunar descent vehicle may contain some uncertainties. Similarly, navigation errors may occur during the end of powered descent to determine the appropriate value of the final velocity of the landing spacecraft. Therefore, in this study, the uncertainties of the vehicle's initial and final velocity are considered to confirm the robustness of the proposed scheme.

Again, due to the attitude error, the initial and final velocity vector pitch angle may contain uncertainties. It is one of the major parameters of the proposed reference trajectory generation scheme and crucial to evaluate its uncertainty effect on the robustness of the system. The determination of lunar gravitational acceleration can be affected by the navigational error during the powered descent phase. Therefore, the authors considered the uncertainty for lunar gravitational acceleration to study on the robustness.

To confirm the performance of the proposed advanced analytical trajectory generation algorithm, paths are calculated with the deviation of initial velocity, final velocity, initial velocity vector pitch angle, and lunar gravity. The nominal condition is the same one used in the previous section.

Figures 8-12 show the flight paths for the cases described in Table 1. Terminal landing position is not specified in this research. These figures show how the parameter uncertainties affect the landing position. Increasing the initial velocity increases the horizontal span and vertical range, as shown in Figure 8. The increased uncertainty of lunar gravitational acceleration also increases the horizontal span and vertical range, as shown in Figure 9. Increasing the initial velocity vector pitch angle causes the vertical range to decrease but the horizontal span increases slightly, as shown in Figures 10 and 11. Therefore, the uncertainty of initial velocity vector pitch angle has less effect on horizontal span. The effect of the uncertainty on final velocity is smaller than the other uncertainties, as shown in Figure 12. The numerical results show that the trajectory generation scheme is highly robust. 


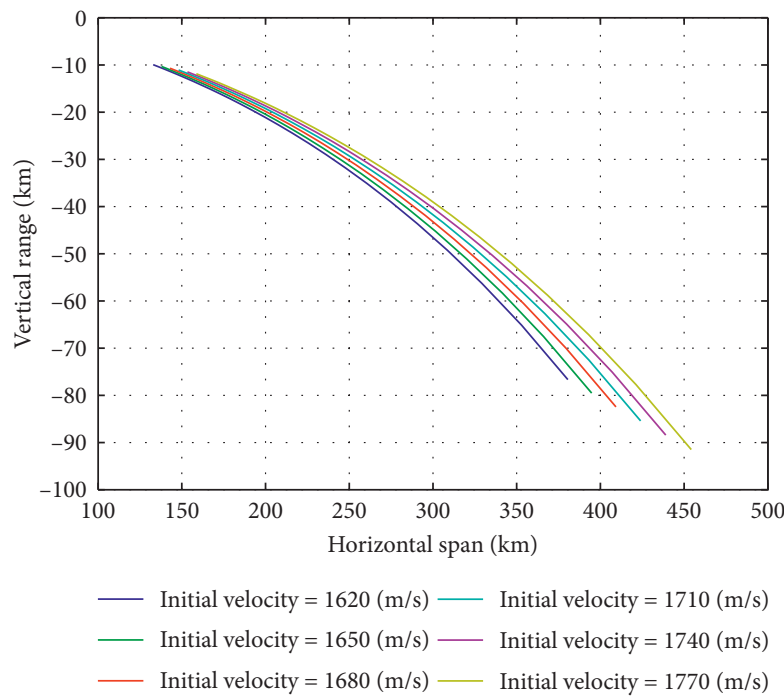

FIGURE 8: Flight path: robustness against initial velocity uncertainty.

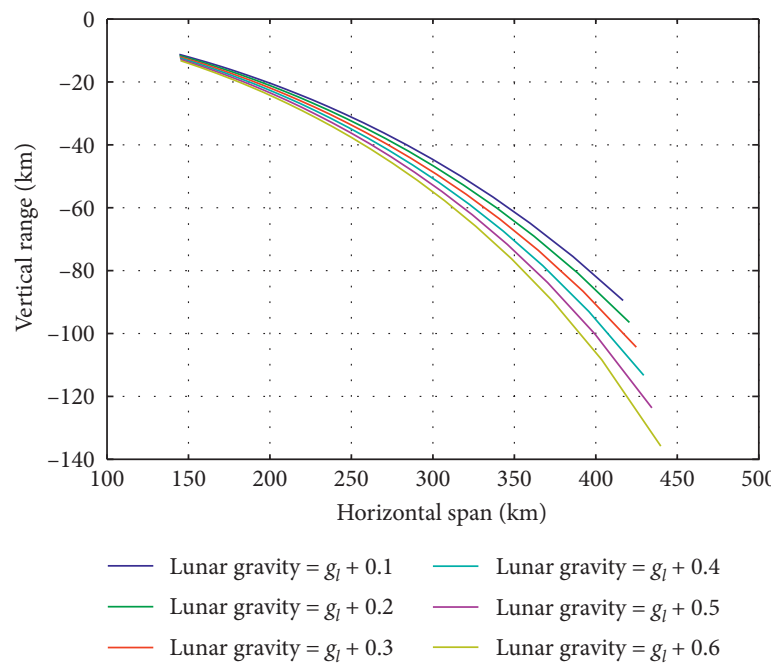

FiguRE 9: Flight path: robustness against lunar gravity uncertainty.

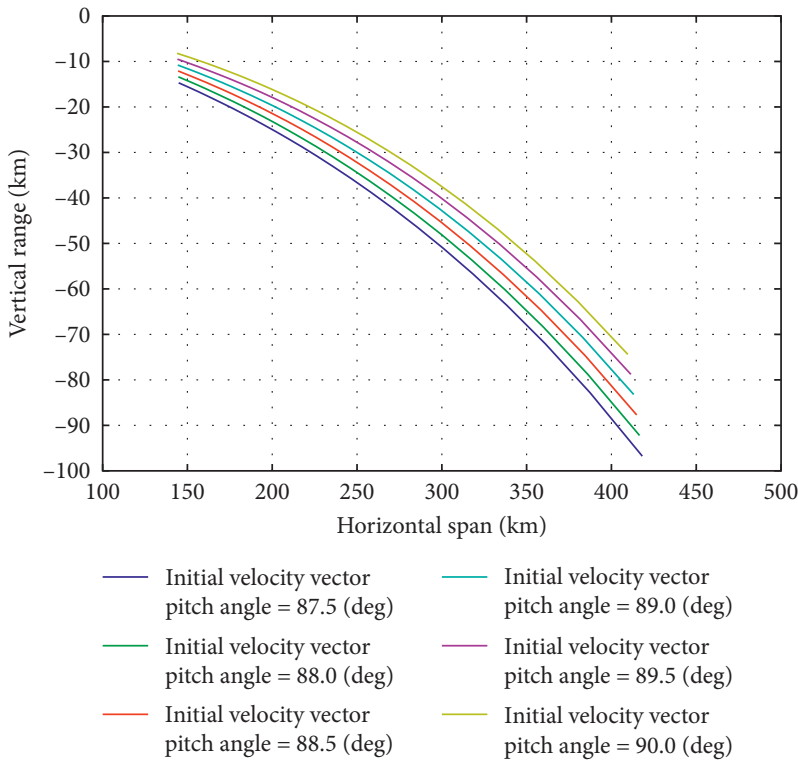

FIGURE 10: Flight path: robustness against initial velocity vector pitch angle uncertainty $\leq 90 \mathrm{deg}$. 


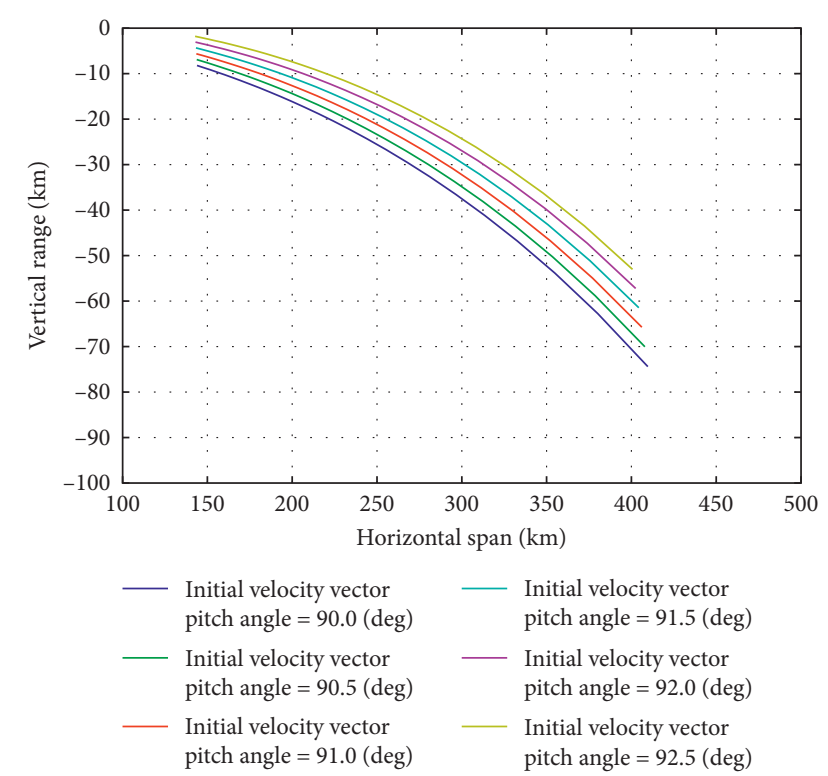

FIGURE 11: Flight path: robustness against initial velocity vector pitch angle uncertainty $\geq 90 \mathrm{deg}$.

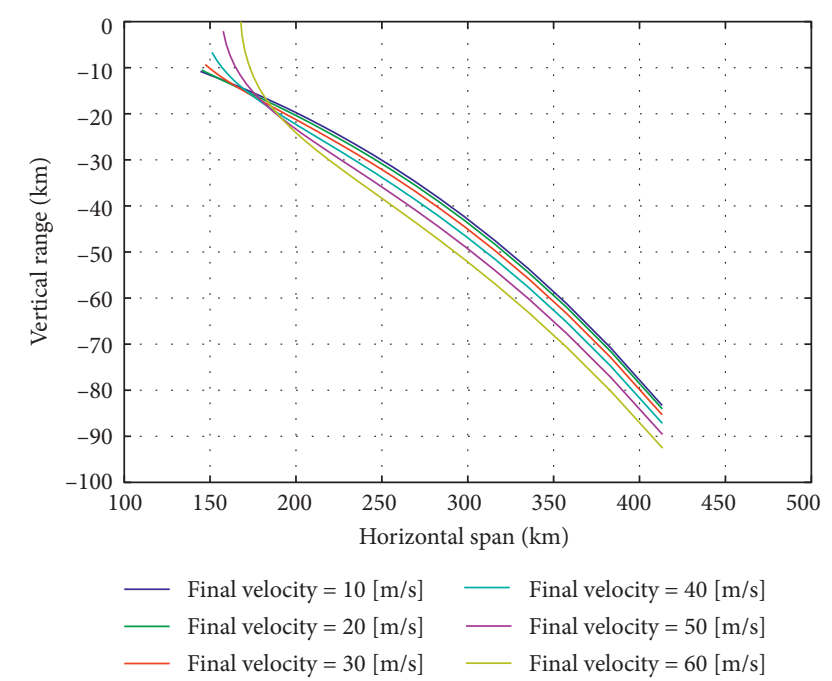

FIGURE 12: Flight path: robustness against final velocity uncertainty.

\section{Conclusion}

To overcome the shortcoming of existing descent schemes and to meet future mission expectations, an advanced descent and trajectory generation scheme is proposed in this study. It is analytical at its basis and can be implemented automatically as demonstrated. The proposed scheme implements a qualitative descent solution to the equation for spacecraft speed, horizontal span, and vertical range as a function of velocity vector pitch angle and has developed an analytical algorithm for dual-step reference trajectory generation. The new proposed scheme satisfies the vertical terminal landing condition to confirm a safe lunar landing mission. Entire descent solutions and comparisons are represented here for both two-dimensional and three-dimensional illuminations. Additionally, an advanced analytical dual-step reference trajectory generation scheme is developed from equations obtained through the advanced lunar descent solution scheme to provide an accurate descent path starting from circular or elliptical parking orbit conditions. A robustness study is also carried out on the proposed reference trajectory generating algorithm. Simulations and analytical studies showed that the proposed schemes were highly effective and useful for the lunar mission.

\section{Data Availability}

No data were used to support this study.

\section{Conflicts of Interest}

The authors declare that they have no conflicts of interest.

\section{Acknowledgments}

This Project was funded by the Deanship of Scientific Research (DSR) at King Abdulaziz University, Jeddah, under grant no. G: 293-135-1440. The authors, therefore, acknowledge with thanks DSR for technical and financial support.

\section{References}

[1] R. K. Cheng, Lunar Terminal Guidance, Wiley, New York, NY, USA, 1964.

[2] R. K. Cheng, D. A. Conrad, and C. M. Meredith, "Design considerations for surveyor guidance," Journal of Spacecraft and Rockets, vol. 3, no. 11, pp. 1569-1576, 1966.

[3] W. Iqbal, H. Hiesinger, and C. H. van der Bogert, "Geological mapping and chronology of lunar landing sites: Apollo 12," Icarus, vol. 352, Article ID 113991, 2020.

[4] A. R. Klumpp, Apollo Guidance, Navigation and Control: Apollo Lunar Descent Guidance, MIT Charles Stark Draper Laboratory, Cambridge, MA, USA.

[5] C. Bei and W. Zhang, "A guidance and control solution for small lunar probe precise-landing mission," Acta Astronautica, vol. 62, no. 1, pp. 44-47, 2008.

[6] S. P. Gong, J. F. Li, H. X. Baoyin, and Y. F. Gao, Lunar Landing Trajectory Design Based on Invariant Manifold, Springer, Berlin, Germany, 2007.

[7] X.-L. Liu, G.-R. Duan, and K.-L. Teo, "Optimal soft landing control for moon lander," Automatica, vol. 44, no. 4, pp. 1097-1103, 2008.

[8] C. R. McInnes, "Gravity-turn descent from low circular orbit conditions," Journal of Guidance, Control, and Dynamics, vol. 26, no. 1, pp. 183-185, 2003.

[9] R. Sostaric, Lunar Descent Reference Trajectory, NASA, JSC, Washington, DC, USA, 2006.

[10] S. Ueno and Y. Yamaguchi, "Near-minimum guidance law of a lunar landing module," 2003.

[11] M. Chloe, P. Virginie, and M. Francesco, "Magma ascent at floor-fractured craters diagnoses the lithospheric stress state on the moon," Earth and Planetary Science Letters, vol. 530, Article ID 115889, 2020. 
[12] P. R. Kumaresan, J. Saravanavel, and K. Palanivel, "Lithological mapping of eratosthenes crater region using moon mineralogy mapper of chandrayaan-1," Planetary and Space Science, vol. 182, Article ID 104817, 2020.

[13] D. J. Scheeres, "Interactions between ground-based and autonomous navigation for precision landing at small solarsystem bodies," Telecommunication and Data Acquisition Progress Report, vol. 42, no. 132, pp. 1-12, 1998.

[14] N. Remesh, R. V. Ramanan, and V. R. Lalithambika, "Fueloptimal and energy-optimal guidance schemes for lunar soft landing at a desired location," Advances in Space Research, vol. 67, no. 6, pp. 1787-1804, 2021.

[15] K. Uchiyama, Y. Shimada, and S. Nakajima, "Tracking control to near optimal trajectory for a lunar lander," in Proceedings of the 23rd International Symposium on Space Technology and Science, vol. 1, pp. 977-982, Barcelona, Spain, December 2002.

[16] C. R. McInnes, "Direct adaptive control for gravity-turn descent," Journal of Guidance, Control, and Dynamics, vol. 22, no. 2, pp. 373-375, 1999.

[17] I. M. Mehedi and T. Kubota, "Advanced guidance scheme for lunar descent and landing from orbital speed conditions," Transaction of Japan Society for Aeronautical and Space Sciences, vol. 54, no. 184, pp. 99-105, 2011.

[18] I. M. Mehedi, T. Kubota, and U. M. Al-Saggaf, "3- dimensional analytical solution for lunar descent scheme," Iranian Journal of Science and Technology Transactions of Electrical Engineering, vol. 39, no. E1, pp. 67-77, 2015.

[19] I. M. Mehedi, T. Kubota, and U. M. Al-Saggaf, "3- dimensional advanced solution of lunar descent and landing," Journal of Vibroengineering, vol. 17, no. 1, pp. 527-543, 2015.

[20] I. M. Mehedi and M. S. Islam, "Moon landing trajectory optimization," International Journal of Advanced Computer Science and Applications, vol. 7, no. 3, pp. 208-286, 2016. 